\title{
Construction of fixed points of asymptotically nonexpansive mappings in uniformly convex hyperbolic spaces
}

\author{
Andrei Sipoşa,b \\ ${ }^{a}$ Research Center for Logic, Optimization and Security (LOS), Department of Computer Science, \\ Faculty of Mathematics and Computer Science, University of Bucharest, \\ Academiei 14, 010014 Bucharest, Romania \\ ${ }^{b}$ Simion Stoilow Institute of Mathematics of the Romanian Academy, \\ Calea Griviței 21, 010702 Bucharest, Romania \\ E-mail: andrei.sipos@fmi.unibuc.ro
}

\begin{abstract}
Kohlenbach and Leuştean have shown in 2010 that any asymptotically nonexpansive self-mapping of a bounded nonempty $U C W$-hyperbolic space has a fixed point. In this paper, we adapt a construction due to Moloney in order to provide a sequence that converges strongly to such a fixed point.

Mathematics Subject Classification 2010: 47H09, 47H10, 47J25.

Keywords: Hyperbolic spaces, uniformly convex hyperbolic spaces, asymptotically nonexpansive mappings, fixed points.
\end{abstract}

\section{Introduction}

In 1972, Goebel and Kirk generalized [4] the classical Browder-Göhde-Kirk theorem to the class of asymptotically nonexpansive mappings (also introduced in that paper), which are mappings $T$ having the property that there is a $\left(k_{n}\right) \subseteq[0, \infty)$ with $\lim _{n \rightarrow \infty} k_{n}=0$ such that for any $x, y$ in the domain of $T$ and any $n \in \mathbb{N}$,

$$
d\left(T^{n} x, T^{n} y\right) \leq\left(1+k_{n}\right) d(x, y),
$$

i.e. they showed that any self-mapping of a bounded closed convex nonempty subset of a uniformly convex Banach space with the above property has a fixed point.

In the recent decades, there has been a renewed interest in fixed point theory and convex optimization as practiced in nonlinear generalizations of the classical structures of functional analysis. For example, there exists in the literature a number of definitions of a notion of a 'hyperbolic space' [2, 5, 6, 9, 19, 20], that aim to axiomatize the convexity structure of normed spaces. The kind of spaces that we shall employ here have a particularly flexible definition due to Kohlenbach [10] and are called $W$-hyperbolic spaces (see the next section for a definition and [11, pp. 384-387] for a detailed discussion on the relationship between various definitions of hyperbolicity).

Uniform convexity, a property originally due to Clarkson [3], was generalized to this hyperbolic setting (following [6, p. 105]) by Leuştean in [14]. The subclass that is the most natural generalization of uniformly convex Banach spaces has then been identified with the one having a monotone modulus of uniform convexity. Those spaces have been called $U C W$-hyperbolic spaces in [15] (see also [16]), where the corresponding Browder-Göhde-Kirk result was proven for spaces of this kind which are complete and nonempty. The Goebel-Kirk extension mentioned above for asymptotically nonexpansive mappings was obtained in the same setting by Kohlenbach and Leuştean in [13].

Thirty years ago, Moloney showed [17, 18] (by refining an earlier result of Kaniel [8]) how to explicitly construct, for any asymptotically nonexpansive self-mapping of a bounded closed convex nonempty subset of a uniformly convex Banach space, a sequence that converges strongly to one of its fixed points. What 
we do in this paper is to show that this construction may be adapted to also work in a bounded nonempty $U C W$-hyperbolic space.

\section{Facts on hyperbolic spaces}

As stated in the Introduction, the following definition is due to Kohlenbach [10].

Definition 2.1. A $W$-hyperbolic space is a triple $(X, d, W)$ where $(X, d)$ is a metric space and $W: X^{2} \times[0,1] \rightarrow X$ such that for all $x, y, z, w \in X$ and $\lambda, \mu \in[0,1]$, we have that

(W1) $d(z, W(x, y, \lambda)) \leq(1-\lambda) d(z, x)+\lambda d(z, y)$;

(W2) $d(W(x, y, \lambda), W(x, y, \mu)=|\lambda-\mu| d(x, y)$;

(W3) $W(x, y, \lambda)=W(y, x, 1-\lambda)$;

(W4) $d(W(x, z, \lambda), W(y, w, \lambda)) \leq(1-\lambda) d(x, y)+\lambda d(z, w)$.

Clearly, any normed space may be made into a $W$-hyperbolic space in a canonical way. As per $[10,14]$, a particular nonlinear class of $W$-hyperbolic spaces is the one of CAT $(0)$ spaces, introduced by A. Aleksandrov [1] and named as such by M. Gromov [7].

A subset $C$ of a $W$-hyperbolic space $(X, d, W)$ is called convex if for any $x, y \in C$ and $\lambda \in[0,1]$, $W(x, y, \lambda) \in C$. If $(X, d, W)$ is a $W$-hyperbolic space, $x, y \in X$ and $\lambda \in[0,1]$, we denote the point $W(x, y, \lambda)$ by $(1-\lambda) x+\lambda y$. We will mainly write $\frac{x+y}{2}$ for $\frac{1}{2} x+\frac{1}{2} y$. The following properties are immediate consequences of the definition of a $W$-hyperbolic space.

Proposition 2.2. Let $(X, d, W)$ be a $W$-hyperbolic space. Let $x, y \in X$ and $\lambda \in[0,1]$. Then we have:

(i) $1 x+0 y=x$;

(ii) $0 x+1 y=y$;

(iii) $(1-\lambda) x+\lambda x=x$;

(iv) $d(x,(1-\lambda) x+\lambda y)=\lambda d(x, y)$;

(v) $d(y,(1-\lambda) x+\lambda y)=(1-\lambda) d(x, y)$.

Definition 2.3. If $(X, d, W)$ is a $W$-hyperbolic space, then a modulus of uniform convexity for $(X, d, W)$ is a function $\eta:(0, \infty) \times(0, \infty) \rightarrow(0,1]$ such that for any $r, \varepsilon>0$ and any $a, x, y \in X$ with $d(x, a) \leq r, d(y, a) \leq r, d(x, y) \geq \varepsilon r$ we have that

$$
d\left(\frac{x+y}{2}, a\right) \leq(1-\eta(r, \varepsilon)) r .
$$

We call the modulus monotone if for any $r, s, \varepsilon>0$ with $s \leq r$, we have $\eta(r, \varepsilon) \leq \eta(s, \varepsilon)$.

Definition 2.4. A $U C W$-hyperbolic space is a quadruple $(X, d, W, \eta)$ where $(X, d, W)$ is a $W$-hyperbolic space and $\eta$ is a monotone modulus of uniform convexity for $(X, d, W)$.

As remarked in [14, Proposition 2.6], CAT(0) spaces are $U C W$-hyperbolic spaces having as a modulus of uniform convexity the function $(r, \varepsilon) \mapsto \frac{\varepsilon^{2}}{8}$, quadratic in $\varepsilon$. Note that a closed convex nonempty subset of a (complete) $(U C) W$-hyperbolic space is itself a (complete) nonempty $(U C) W$-hyperbolic space (in contrast to e.g. normed spaces).

The following is an adaptation of a result due to Kohlenbach and Leuştean, namely [12, Lemma 3.2].

Proposition 2.5. Let $(X, d, W, \eta)$ be a $U C W$-hyperbolic space. Define, for any $r, \varepsilon>0, u(r, \varepsilon):=$ $\frac{\varepsilon}{2} \cdot \eta(r, \varepsilon)$. Then, for any $r, \varepsilon>0$ and any $a, x, y \in X$ with $d(x, a) \leq d(y, a) \leq r$ and $d(x, y) \geq \varepsilon r$ we have that

$$
d\left(\frac{x+y}{2}, a\right) \leq d(y, a)-u(r, \varepsilon) r .
$$

In addition, if there is a function $\eta^{\prime}$ which is nondecreasing in its second argument such that for all $r$ and $\varepsilon, \eta(r, \varepsilon)=\varepsilon \eta^{\prime}(r, \varepsilon)$ (e.g. in the case of $C A T(0)$ spaces, as per the above remark), then one can take $u$ to be simply $\eta$. 
Proof. Let $r, \varepsilon>0$ and $a, x, y \in X$ be as required. First, note that

$$
\frac{\varepsilon r}{2} \leq \frac{d(x, y)}{2} \leq \frac{d(x, a)+d(y, a)}{2} \leq d(y, a),
$$

so, using that $\eta$ is a monotone modulus of uniform convexity, we get that

$d\left(\frac{x+y}{2}, a\right) \leq(1-\eta(d(y, a), \varepsilon)) \cdot d(y, a) \leq(1-\eta(r, \varepsilon)) \cdot d(y, a) \leq d(y, a)-\eta(r, a) \frac{\varepsilon r}{2}=d(y, a)-u(r, \varepsilon) r$.

The hypotheses imply that $d(y, a) \neq 0$, so we may write

$$
d(x, y) \geq \varepsilon r=\frac{\varepsilon r}{d(y, a)} \cdot d(y, a),
$$

and therefore, in the second case,

$$
\begin{aligned}
d\left(\frac{x+y}{2}, a\right) & \leq\left(1-\eta\left(d(y, a), \frac{\varepsilon r}{d(y, a)}\right)\right) \cdot d(y, a)=d(y, a)-d(y, a) \cdot \frac{\varepsilon r}{d(y, a)} \cdot \eta^{\prime}\left(d(y, a), \frac{\varepsilon r}{d(y, a)}\right) \\
& =d(y, a)-\varepsilon r \eta^{\prime}\left(d(y, a), \frac{\varepsilon r}{d(y, a)}\right) \leq d(y, a)-\varepsilon r \eta^{\prime}(d(y, a), \varepsilon) \\
& =d(y, a)-r \eta(d(y, a), \varepsilon) \leq d(y, a)-\eta(r, \varepsilon) r .
\end{aligned}
$$

Definition 2.6. Let $(X, d)$ be a metric space, $T: X \rightarrow X$ and $\left(k_{n}\right) \subseteq[0, \infty)$ such that $\lim _{n \rightarrow \infty} k_{n}=0$. Then $T$ is called asymptotically nonexpansive with respect to $\left(k_{n}\right)$ if for any $x, y \in X$ and any $n \in \mathbb{N}$,

$$
d\left(T^{n} x, T^{n} y\right) \leq\left(1+k_{n}\right) d(x, y) .
$$

For any self-mapping $T$ (of an arbitrary set), we denote the set of its fixed points by Fix(T). In [13], Kohlenbach and Leustean have proved that any asymptotically nonexpansive self-mapping of a bounded complete nonempty $U C W$-hyperbolic space has a fixed point.

\section{Main results}

We fix a complete nonempty $U C W$-hyperbolic space $(X, d, W, \eta)$ and $b>0$ an upper bound for its diameter. Let $\left(k_{n}\right) \subseteq[0, \infty)$ be such that $\lim _{n \rightarrow \infty} k_{n}=0$ and $T: X \rightarrow X$ be asymptotically nonexpansive with respect to $\left(k_{n}\right)$, so $\operatorname{Fix}(T) \neq \emptyset$.

We shall construct a mapping $S: X \rightarrow X$ such that:

(i) $\operatorname{Fix}(T)=\operatorname{Fix}(S)($ so $\operatorname{Fix}(S) \neq \emptyset)$;

(ii) for any $p \in F i x(S)$ and $x \in X, d(S x, p) \leq d(x, p)$;

(iii) for any $\left(x_{n}\right) \subseteq X$ having $x \in X$ as its limit and with $\lim _{n \rightarrow \infty} d\left(x_{n}, S x_{n}\right)=0$, we have $x \in \operatorname{Fix}(S)$.

Note that if $T$ is nonexpansive we may simply take $S:=T$.

Lemma 3.1. Let $x \in X$. Then for any $n \in \mathbb{N}$ there is an $m \in\{n, n+1\}$ such that

$$
d\left(T^{m} x, x\right) \geq \frac{1}{2+k_{1}} d(T x, x) .
$$

Proof. Let $n \in \mathbb{N}$. Assume by way of contradiction that

$$
d\left(T^{n} x, x\right)<\frac{1}{2+k_{1}} d(T x, x)
$$

and

$$
d\left(T^{n+1} x, x\right)<\frac{1}{2+k_{1}} d(T x, x) .
$$


Then, since

$$
d\left(T^{n+1} x, T x\right) \leq\left(1+k_{1}\right) d\left(T^{n} x, x\right)<\frac{1+k_{1}}{2+k_{1}} d(T x, x)
$$

we have that

$$
d(T x, x) \leq d\left(T^{n+1} x, x\right)+d\left(T^{n+1} x, T x\right)<\frac{1}{2+k_{1}} d(T x, x)+\frac{1+k_{1}}{2+k_{1}} d(T x, x)=d(T x, x),
$$

a contradiction.

Let now $x \in X$. If $T x=x$, put $S x:=x$. If $T x \neq x$, then put $n$ be minimal such that $k_{n}$ and $k_{n+1}$ are both smaller or equal than

$$
\min \left(\frac{d(x, T x)}{2 b}, 2 \eta\left(b, \frac{d(x, T x)}{2 b}\right)\right)>0
$$

(such an $n$ exists since $\lim _{n \rightarrow \infty} k_{n}=0$ ). Then, making use of Lemma 3.1, put $m \in\{n, n+1\}$ be minimal such that

$$
d\left(T^{m} x, x\right) \geq \frac{1}{2+k_{1}} d(T x, x)
$$

and set

$$
S x:=\frac{T^{m} x+x}{2} .
$$

The following proposition shows that $S$ has all the required properties.

Proposition 3.2. Let $x \in X, p \in F i x(S)$ and $\left(x_{n}\right) \subseteq X$. We have that:

(i) $d(S x, x) \geq \frac{1}{2\left(2+k_{1}\right)} d(T x, x)$;

(ii) $\operatorname{Fix}(T)=\operatorname{Fix}(S)$;

(iii) $d(S x, p) \leq d(x, p)$;

(iv) if $x$ is the limit of $\left(x_{n}\right)$ and $\lim _{n \rightarrow \infty} d\left(x_{n}, S x_{n}\right)=0$, we have $x \in \operatorname{Fix}(S)$.

Proof. (i) If $T x=x$, there is nothing to show. If $T x \neq x$, then, by putting $m$ to be the one from the construction of $S x$, we have that $S x=\frac{T^{m} x+x}{2}$, so $d(S x, x)=\frac{1}{2} d\left(T^{m} x, x\right) \geq \frac{1}{2\left(2+k_{1}\right)} d(T x, x)$.

(ii) The inclusion $F i x(T) \subseteq F i x(S)$ follows by the construction of $S ; F i x(S) \subseteq F i x(T)$ follows by (i).

(iii) We have that $p \in F i x(T)$. If $T x=x$, there is nothing to show. Suppose, then, that $T x \neq x$, so, again by putting $m$ to be the one from the construction of $S x$, we have that $S x=\frac{T^{m} x+x}{2}$.

Put $c:=\frac{1}{\left(2+k_{1}\right)} d(T x, x), q:=d(x, p)$ and $\varepsilon:=\frac{c}{2 b}$. Since $m$ was chosen such that $k_{m} \leq \varepsilon$, we have that

$$
b\left(k_{m}+\varepsilon\right) \leq 2 b \varepsilon=c .
$$

Therefore, since $q \leq b$,

$$
d\left(T^{m} x, x\right) \geq c \geq b\left(k_{m}+\varepsilon\right) \geq q\left(k_{m}+\varepsilon\right),
$$

so $d\left(T^{m} x, x\right)-q k_{m} \geq q \varepsilon$. In addition, using the monotonicity of $\eta$ and (again) the way $m$ was chosen,

$$
\eta(q, \varepsilon) \geq \eta(b, \varepsilon) \geq k_{m} / 2 \text {. }
$$

If $d\left(T^{m} x, p\right)<q$, then

$$
d(S x, p)=d\left(\frac{T^{m} x+x}{2}, p\right) \leq \frac{1}{2} d\left(T^{m} x, p\right)+\frac{1}{2} d(x, p) \leq q .
$$

Consider now the case where $d\left(T^{m} x, p\right) \geq q$. Then $d\left(T^{m} x, p\right) \leq\left(1+k_{m}\right) d(x, p)=\left(1+k_{m}\right) q$, so there is a $\beta \in\left[0, k_{m}\right]$ with $d\left(T^{m} x, p\right)=(1+\beta) q$. Put

$$
y:=\frac{1}{1+\beta} T^{m} x+\frac{\beta}{1+\beta} p .
$$


Then

$$
d\left(T^{m} x, y\right)=\frac{\beta}{1+\beta} d\left(T^{m} x, p\right)=q \beta \leq q k_{m},
$$

So

$$
d\left(T^{m} x, x\right) \leq d(x, y)+d\left(T^{m} x, y\right)=d(x, y)+q k_{m}
$$

and thus

$$
d(x, y) \geq d\left(T^{m} x, x\right)-q k_{m} \geq q \varepsilon .
$$

On the other hand, we have that $d(x, p)=q$ and

$$
d(y, p)=\frac{1}{1+\beta} d\left(T^{m} x, p\right)=q,
$$

so, since $\eta$ is a modulus of uniform convexity,

$$
d\left(\frac{x+y}{2}, p\right) \leq(1-\eta(q, \varepsilon)) q .
$$

Then

$$
\begin{aligned}
d(S x, p) & =d\left(\frac{T^{m} x+x}{2}, p\right) \leq d\left(\frac{x+y}{2}, p\right)+d\left(\frac{x+y}{2}, \frac{x+T^{m} x}{2}\right) \\
& \leq(1-\eta(q, \varepsilon)) q+\frac{1}{2} d\left(T^{m} x, y\right) \leq\left(1-\frac{k_{m}}{2}\right) q+\frac{q k_{m}}{2}=q
\end{aligned}
$$

(iv) From (i), we get that $\lim _{n \rightarrow \infty} d\left(x_{n}, T x_{n}\right)=0$, then, by the continuity of $T$, we get $x \in F i x(T)=$ $\operatorname{Fix}(S)$.

Given $S$ with these properties and $x \in X$, we will now construct a sequence converging to a fixed point of $S$.

For any $j \geq 1$, we shall set an $m_{j} \geq 1$ and a finite sequence $\left(z_{i j}\right)_{i=1}^{m_{j}}$, and we shall put $y_{j}:=z_{m_{j} j}$. The sequence $\left(y_{j}\right)$ will be the one we are after.

Put $m_{1}:=1$ and $z_{11}:=x$. Assume now that we have constructed the $j$ th finite sequence and we are seeking the next one. We distinguish two cases.

Construction case $\mathbf{I}$. There is an $i \in\left[2, m_{j}-1\right]$ such that $d\left(z_{i j}, z_{(i+1) j}\right)<d\left(z_{i j}, z_{(i-1) j}\right)$ and $d\left(z_{i j}, \frac{z_{i j}+S z_{m_{j} j}}{2}\right) \geq d\left(z_{i j}, z_{(i-1) j}\right)$.

Let $i$ be minimal with this property. Then put $m_{j+1}:=i+1$, put for all $k \leq i, z_{k(j+1)}:=z_{k j}$ and $y_{j+1}=z_{(i+1)(j+1)}:=\frac{z_{i j}+S z_{m_{j} j}}{2}$.

Construction case II. There is no such $i$.

In this case, put $m_{j+1}:=m_{j}+1$, put for all $k \leq m_{j}, z_{k(j+1)}:=z_{k j}$ and $z_{\left(m_{j}+1\right)(j+1)}:=\frac{z_{m_{j} j}+S z_{m_{j} j}}{2}$.

It is immediate that $m_{2}=2$ and $m_{3}=3$. By a simple induction, it follows that for all $j \geq 3, m_{j} \geq 3$.

Lemma 3.3. Let $p \in F i x(S), j \in \mathbb{N}$ and $i \in\left[1, m_{j}-1\right]$. Then $d\left(z_{(i+1) j}, p\right) \leq d\left(z_{i j}, p\right)$.

Proof. We prove this by induction on $j$. If $j=1$, the property holds vacuously. Suppose now that the property holds for $j$ and we want to prove it for $j+1$. If $i<m_{j+1}-1, z_{(i+1)(j+1)}=z_{(i+1) j}$ and $z_{i(j+1)}=z_{i j}$, so we simply apply the induction hypothesis. If $i=m_{j+1}-1$, then $z_{i(j+1)}=z_{i j}$ and $z_{(i+1)(j+1)}=\frac{z_{i j}+S z_{m_{j} j}}{2}$, so

$$
d\left(z_{(i+1)(j+1)}, p\right)=d\left(\frac{z_{i j}+S z_{m_{j} j}}{2}, p\right) \leq \frac{1}{2} d\left(z_{i j}, p\right)+\frac{1}{2} d\left(S z_{m_{j} j}, p\right) \leq \frac{1}{2} d\left(z_{i j}, p\right)+\frac{1}{2} d\left(z_{m_{j} j}, p\right)
$$




$$
\leq \frac{1}{2} d\left(z_{i j}, p\right)+\frac{1}{2} d\left(z_{i j}, p\right)=d\left(z_{i j}, p\right)=d\left(z_{i(j+1)}, p\right) .
$$

Lemma 3.4. Let $p \in$ Fix $(S), \varepsilon>0, j \in \mathbb{N}$ and $i \in\left[1, m_{j}-1\right]$. Let $u$ be such that the property described by Proposition 2.5 holds. Assume that $d\left(z_{i j}, z_{(i+1) j}\right) \geq \varepsilon$. Then $d\left(z_{i j}, p\right)-d\left(z_{(i+1) j}, p\right) \geq u\left(b, \frac{\varepsilon}{b}\right) b$.

Proof. We prove this by induction on $j$. If $j=1$, the property holds vacuously. Suppose now that the property holds for $j$ and we want to prove it for $j+1$. If $i<m_{j+1}-1, z_{(i+1)(j+1)}=z_{(i+1) j}$ and $z_{i(j+1)}=z_{i j}$, so we simply apply the induction hypothesis. If $i=m_{j+1}-1$, then $z_{i(j+1)}=z_{i j}$ and $z_{(i+1)(j+1)}=\frac{z_{i j}+S z_{m_{j} j}}{2}$. Using Lemma 3.3, we have that

$$
d\left(S z_{m_{j} j}, p\right) \leq d\left(z_{m_{j} j}, p\right) \leq d\left(z_{i j}, p\right) \leq b .
$$

In addition,

$$
d\left(z_{i j}, S z_{m_{j} j}\right) \geq \frac{d\left(z_{i j}, S z_{m_{j} j}\right)}{2}=d\left(z_{i j}, z_{(i+1)(j+1)}\right)=d\left(z_{i(j+1)}, z_{(i+1)(j+1)}\right) \geq \varepsilon=\frac{\varepsilon}{b} \cdot b,
$$

so, applying Proposition 2.5,

$$
d\left(z_{(i+1)(j+1)}, p\right) \leq d\left(z_{i(j+1)}, p\right)-u\left(b, \frac{\varepsilon}{b}\right) b .
$$

We shall now construct a sequence $p_{1}<p_{2}<\ldots$ such that for any $k \geq 1, m_{p_{k}}=k$ (so $z_{k p_{k}}=y_{p_{k}}$ ) and for all $j \geq p_{k}+1, m_{j} \geq k+1$ and $z_{k j}=z_{k p_{k}}$, and $p_{k}$ is optimal in this regard, i.e. either $z_{k\left(p_{k}-1\right)}$ is not defined or $z_{k\left(p_{k}-1\right)} \neq z_{k p_{k}}$ (which makes it uniquely determined). We shall denote, for all $k \geq 1$, $x_{k}:=y_{p_{k}}$. We will also show that for all $k, d\left(x_{k}, x_{k+1}\right) \geq d\left(x_{k}, \frac{x_{k}+S x_{k}}{2}\right)$, i.e. $2 d\left(x_{k}, x_{k+1}\right) \geq d\left(x_{k}, S x_{k}\right)$.

It is clear that one must have $p_{1}:=1\left(\right.$ so $\left.x_{1}=x\right)$. Assume that we have constructed the sequence up to $p_{k}$ and we want to find the value of $p_{k+1}$.

We know that $m_{p_{k}+1} \geq k+1$, but since $m_{p_{k}+1} \leq m_{p_{k}}+1=k+1, m_{p_{k}+1}=k+1=m_{p_{k}}+1$. Thus the $\left(p_{k}+1\right)$ th line was obtained using Construction case II, so

$$
z_{(k+1)\left(p_{k}+1\right)}=\frac{x_{k}+S x_{k}}{2} .
$$

In the case where for all $t \geq p_{k}+1, z_{(k+1) t}=z_{(k+1)\left(p_{k}+1\right)}$, in order to simply put $p_{k+1}:=p_{k}+1$, we must also show that for all $j \geq p_{k}+2, m_{j} \geq k+2$. Assume that there is a $j \geq p_{k}+2$ with $m_{j}<k+2$, i.e. $m_{j}=k+1$. Since $m_{j-1} \geq k+1$, the $j$ th sequence must have necessarily been obtained via Construction case I with $i=k$, so

$$
\begin{gathered}
d\left(z_{k(j-1)}, z_{(k+1)(j-1)}\right)<d\left(z_{k(j-1)}, z_{(k-1)(j-1)}\right), \\
d\left(z_{k(j-1)}, \frac{z_{k(j-1)}+S z_{m_{j-1}(j-1)}}{2}\right) \geq d\left(z_{k(j-1)}, z_{(k-1)(j-1)}\right),
\end{gathered}
$$

and $z_{(k+1) j}=\frac{z_{k(j-1)}+S z_{m_{j-1}(j-1)}}{2}$. Since, by our assumption, $z_{(k+1) j}=z_{(k+1)\left(p_{k}+1\right)}=z_{(k+1)(j-1)}$, we have that (2) yields

$$
d\left(z_{k(j-1)}, z_{(k+1)(j-1)}\right) \geq d\left(z_{k(j-1)}, z_{(k-1)(j-1)}\right),
$$

which contradicts (1). In this case $x_{k+1}=z_{(k+1)\left(p_{k}+1\right)}=\frac{x_{k}+S x_{k}}{2}$, so $d\left(x_{k}, x_{k+1}\right)=d\left(x_{k}, \frac{x_{k}+S x_{k}}{2}\right)$.

Assume now that there is a $t \geq p_{k}+2$ with $z_{(k+1) t} \neq z_{(k+1)\left(p_{k}+1\right)}$ and take it to be minimal ( $a$ posteriori it will be unique). Then, since $m_{t-1} \geq k+1$, we have that the $t$ th sequence must have been obtained via Construction case I with $i=k$, so $m_{t}=k+1$,

$$
d\left(z_{k(t-1)}, z_{(k+1)(t-1)}\right) \geq d\left(z_{k(t-1)}, z_{(k-1)(t-1)}\right)
$$

and

$$
d\left(z_{k(t-1)}, \frac{z_{k(t-1)}+S z_{m_{t-1}(t-1)}}{2}\right) \geq d\left(z_{k(t-1)}, z_{(k-1)(t-1)}\right) .
$$


For all $s \leq k, t-1 \geq p_{k} \geq p_{s}$, so $z_{s t}=z_{s(t-1)}$, and since $z_{(k+1) t}=\frac{z_{k(t-1)}+S z_{m_{t-1}(t-1)}}{2}$, (4) yields

$$
d\left(z_{k t}, z_{(k+1) t}\right) \geq d\left(z_{k t}, z_{(k-1) t}\right) .
$$

We will now show that for all $j \geq t+1, m_{j} \geq k+2$ and $z_{(k+1) j}=z_{(k+1) t}$, so we may put $p_{k+1}:=t$. Start with $j:=t+1$. Suppose that $m_{t+1}<k+2$, i.e. $m_{t+1}=k+1$. Then the $(t+1)$ th sequence must have been obtained via Construction case I with $i=k$, so $d\left(z_{k t}, z_{(k+1) t}\right)<d\left(z_{k t}, z_{(k-1) t}\right)$, which contradicts (5). Since then $m_{t+1} \geq k+2=m_{t}+1$, the $(t+1)$ th sequence must have been obtained via Construction case II, so $z_{(k+1)(t+1)}=z_{(k+1) t}$. Assume now that the property to be proven holds for the $j$ th sequence and we want to prove it for the next one. By the induction hypothesis, $z_{(k+1) j}=z_{(k+1) t}$. Suppose that $m_{j+1}<k+2$, i.e. $m_{j+1}=k+1$. Then the $(j+1)$ th sequence must have been obtained via Construction case I with $i=k$, so

$$
d\left(z_{k j}, z_{(k+1) j}\right)<d\left(z_{k j}, z_{(k-1) j}\right) .
$$

We have that for all $s \leq k, j \geq p_{k} \geq p_{s}$, so $z_{s j}=z_{s t}$, and since, as stated before, $z_{(k+1) j}=z_{(k+1) t}$, (6) yields that $d\left(z_{k t}, z_{(k+1) t}\right)<d\left(z_{k t}, z_{(k-1) t}\right)$, which contradicts $(5)$. Since then $m_{j+1} \geq k+2$, the $(j+1)$ th sequence must have been obtained via Construction case I with $i \geq k+1$ or via Construction case II, so $z_{(k+1)(j+1)}=z_{(k+1) j}=z_{(k+1) t}$. In addition, by the minimality of $t$, we get that

$$
z_{(k+1)(t-1)}=z_{(k+1)\left(p_{k}+1\right)}=\frac{x_{k}+S x_{k}}{2},
$$

so (3) yields that

$$
d\left(x_{k}, \frac{x_{k}+S x_{k}}{2}\right)<d\left(x_{k}, x_{k-1}\right)
$$

while (5) means that

$$
d\left(x_{k}, x_{k+1}\right) \geq d\left(x_{k}, x_{k-1}\right),
$$

SO

$$
d\left(x_{k}, x_{k+1}\right)>d\left(x_{k}, \frac{x_{k}+S x_{k}}{2}\right) .
$$

We have now finished constructing the $p_{k}$ 's.

Lemma 3.5. Let $p \in \operatorname{Fix}(S)$ and $k \in \mathbb{N}$. Then for all $n \geq p_{k}, d\left(y_{n}, p\right) \leq d\left(x_{k}, p\right)$.

Proof. Let $n \geq p_{k}$, so $m_{n} \geq k$. By Lemma $3.3, d\left(z_{m_{n} n}, p\right) \leq d\left(z_{k n}, p\right)$. On the other hand $z_{m_{n} n}=y_{n}$ and since $n \geq p_{k}, z_{k n}=z_{k p_{k}}=x_{k}$, so the conclusion follows.

Lemma 3.6. Let $p \in F i x(S), \varepsilon>0$ and $i \in \mathbb{N}$. Let $u$ be such that the property described by Proposition 2.5 holds. Assume that $d\left(x_{i}, x_{i+1}\right) \geq \varepsilon$. Then $d\left(x_{i}, p\right)-d\left(x_{i+1}, p\right) \geq u\left(b, \frac{\varepsilon}{b}\right) b$.

Proof. Set $j:=p_{i+1}$. We have that $m_{j}=m_{p_{i+1}}=i+1$, so $i \in\left[1, m_{j}-1\right]$. Note that $z_{(i+1) j}=$ $z_{(i+1) p_{i+1}}=x_{i+1}$ and that, since $j \geq p_{i}, z_{i j}=z_{i p_{i}}=x_{i}$. Now apply Lemma 3.4.

Lemma 3.7. We have that $\lim _{n \rightarrow \infty} d\left(x_{n}, x_{n+1}\right)=0$.

Proof. Here is where we use that $\operatorname{Fix}(S) \neq \emptyset$. Let $p \in \operatorname{Fix}(S)$. Assume that our conclusion is false, i.e. there is an $\varepsilon>0$ such that for all $N$ there is an $n>N$ such that $d\left(x_{n}, x_{n+1}\right)>\varepsilon$. Denote, for all $n, a_{n}:=d\left(x_{n}, x_{n+1}\right)$ and $c_{n}:=d\left(x_{n}, p\right)-d\left(x_{n+1}, p\right)$. Put $d_{0}:=1$ and for all $n \geq 0$, put $d_{n+1}>d_{n}$ such that $a_{d_{n+1}}>\varepsilon$. In particular, we have that for all $i \geq 1, a_{d_{i}}>\varepsilon$. Let $u$ be such that the property described by Proposition 2.5 holds and set $k:=\left\lceil\frac{b+1}{u\left(b, \frac{\varepsilon}{b}\right) b}\right\rceil$. Applying Lemma 3.6, we get that for all $i \geq 1, c_{d_{i}} \geq u\left(b, \frac{\varepsilon}{b}\right) b$. We may thus write

$$
b \geq d\left(x_{1}, p\right) \geq d\left(x_{1}, p\right)-d\left(x_{d_{k}+1}, p\right)=\sum_{n=1}^{d_{k}} c_{n} \geq \sum_{i=1}^{k} c_{d_{i}} \geq k \cdot u\left(b, \frac{\varepsilon}{b}\right) b \geq b+1,
$$

a contradiction. 
Lemma 3.8. Let $n$ be such that $x_{n}=x_{n+1}=x_{n+2}$. Then:

(i) $x_{n}=S x_{n}$

(ii) for all $j \geq p_{n+2}$ and all $q \in\left[n, m_{j}\right], z_{q j}=x_{n}$;

(iii) for all $j \geq p_{n+2}, y_{j}=x_{n}$;

(iv) for all $q \geq n, x_{q}=x_{n}$.

Proof. (i) Put $j:=p_{n+2}$. Then $j>p_{n+1}$, so $j-1 \geq p_{n+1}>p_{n}$. Then we get that $z_{(n+1)(j-1)}=$ $z_{(n+1) p_{n+1}}=x_{n+1}$ and $z_{n(j-1)}=z_{n p_{n}}=x_{n}$, so, since $x_{n}=x_{n+1}, d\left(z_{(n+1)(j-1)}, z_{n(j-1)}\right)=0$.

Assume that the $j$ th sequence was obtained using Construction case I. Then we must have, since $m_{j}=n+2, d\left(z_{(n+1)(j-1)}, z_{(n+2)(j-1)}\right)<d\left(z_{(n+1)(j-1)}, z_{n(j-1)}\right)=0$, a contradiction. Thus, it was obtained using Construction case II, so

$$
x_{n+2}=z_{(n+2) j}=\frac{z_{(n+1)(j-1)}+S z_{(n+1)(j-1)}}{2}=\frac{x_{n+1}+S x_{n+1}}{2} .
$$

However, $x_{n+1}=x_{n+2}$, so $0=d\left(x_{n+1}, x_{n+2}\right)=d\left(x_{n+1}, S x_{n+1}\right) / 2$. Thus, $x_{n+1}=S x_{n+1}$, i.e. $x_{n}=S x_{n}$.

(ii) It is clear that this holds for $j=p_{n+2}$. Assume that it holds for a $j \geq p_{n+2}-$ since then $j \geq p_{n+1}+1$, $m_{j} \geq n+2$ - and we want to prove it for $j+1-$ since $j+1 \geq p_{n+2}+1, m_{j+1} \geq p_{n+3}$. Thus, the $(j+1)$ th sequence was obtained either using Construction case I with $i \geq n+2$ or using Construction case II. Let $q \in\left[n, m_{j}\right]$. Then either $z_{q(j+1)}=z_{q j}=x_{n}$ (by the induction hypothesis) or

$$
z_{q j}=\frac{z_{(q-1) j}+S z_{m_{j} j}}{2},
$$

but this can happen only if $q-1 \geq n+2$, so (by the induction hypothesis) $z_{(q-1) j}=x_{n}$ and $z_{m_{j} j}=x_{n}$. Since in addition we know that $x_{n}=S x_{n}$, we have that

$$
z_{q j}=\frac{x_{n}+S x_{n}}{2}=x_{n} .
$$

(iii) Let $j \geq p_{n+2}$ and put $q:=m_{j}$ in the above.

(iv) Let $q \geq n+3$. Then $p_{q} \geq p_{n+2}$ and $x_{q}=y_{p_{q}}=x_{n}$.

Lemma 3.9. Let $n \geq 2$ be such that $d\left(x_{n}, x_{n+1}\right)<d\left(x_{n}, x_{n-1}\right)$. Then for all $u \geq p_{n+1}, 2 d\left(x_{n}, x_{n-1}\right) \geq$ $d\left(x_{n}, S y_{v}\right)$

Proof. Let $v \geq p_{n+1}$, so for all $i \in[n-1, n+1], z_{i v}=x_{i}$. Assume that $2 d\left(x_{n}, x_{n-1}\right)<d\left(x_{n}, S y_{v}\right)$, so

$$
d\left(z_{n v}, z_{(n-1) v}\right) \leq \frac{1}{2} d\left(z_{n v}, S z_{m_{v} v}\right)=d\left(z_{n v}, \frac{z_{n v}+S z_{m_{v} v}}{2}\right) .
$$

We also know that $d\left(z_{n v}, z_{(n+1) v}\right)<d\left(z_{n v}, z_{(n-1) v}\right)$, so the $(v+1)$ th sequence is obtained by Construction case I with $i \leq n$, so $m_{v+1} \leq n+1$. On the other hand $v+1 \geq p_{n+1}+1$, so $m_{v+1} \geq n+2$, a contradiction.

Lemma 3.10. Let $n \geq 2$ be such that $d\left(x_{n}, x_{n+1}\right)<d\left(x_{n}, x_{n-1}\right)$. Then for all $q \geq n, 2 d\left(x_{n}, x_{n-1}\right) \geq$ $d\left(x_{n}, x_{q}\right)$.

Proof. Clearly, the conclusion holds for $q=n$ and $q=n+1$. Let $q \geq n+1$ and assume that the conclusion holds for $q$. We want to prove that it also holds for $q+1$. Put $v:=p_{q+1}-1$. Since $v \geq p_{q}$, $z_{q v}=x_{q}$, so

$$
x_{q+1}=\frac{z_{q v}+S y_{v}}{2}=\frac{x_{q}+S y_{v}}{2} .
$$


By the induction hypothesis, we have that $2 d\left(x_{n}, x_{n-1}\right) \geq d\left(x_{n}, x_{q}\right)$ and since $v \geq p_{q} \geq p_{n+1}$, by Lemma 3.9 we have that $2 d\left(x_{n}, x_{n-1}\right) \geq d\left(x_{n}, S y_{v}\right)$, so

$$
d\left(x_{n}, x_{q+1}\right) \leq \frac{1}{2} d\left(x_{n}, x_{q}\right)+\frac{1}{2} d\left(x_{n}, S y_{v}\right) \leq 2 d\left(x_{n}, x_{n-1}\right)
$$

Proposition 3.11. The sequence $\left(x_{n}\right)$ is Cauchy.

Proof. Let $\varepsilon>0$. We want an $M$ such that for all $n, m \geq M, d\left(x_{n}, x_{m}\right) \leq \varepsilon$. Put $\delta:=\frac{\varepsilon}{4}$. By Lemma 3.7, there is an $N$ such that for all $k \geq N, d\left(x_{k}, x_{k+1}\right) \leq \delta$.

Case I. We have that $x_{N}=x_{N+1}=x_{N+2}$.

Then, by Lemma 3.8 , for all $q \geq N, x_{q}=x_{N}$, so we may take $M:=N$.

Case II. There is a $k \in\{N, N+1\}$ such that $x_{k} \neq x_{k+1}$.

Put $\rho:=d\left(x_{k}, x_{k+1}\right)>0$. Again, by Lemma 3.7, there is a $p \geq k+1$ such that $d\left(x_{p}, x_{p+1}\right) \leq \frac{\rho}{2}$, so there is an $M \in[k+1, p]$ such that $d\left(x_{M}, x_{M+1}\right)<d\left(x_{M}, x_{M-1}\right)$. Thus, by Lemma 3.10, for all $q \geq M$, $2 d\left(x_{M}, x_{M-1}\right) \geq d\left(x_{M}, x_{q}\right)$.

On the other hand, since $M \geq k+1, M-1 \geq k \geq N$, so $d\left(x_{M}, x_{M-1}\right) \leq \delta$ and for all $q \geq M$, $d\left(x_{M}, x_{q}\right) \leq 2 \delta$.

Let $n, m \geq M$. Then $d\left(x_{n}, x_{m}\right) \leq d\left(x_{M}, x_{n}\right)+d\left(x_{M}, x_{m}\right) \leq 4 \delta=\varepsilon$.

Since $X$ is complete, $\left(x_{n}\right)$ is convergent. We denote its limit by $p$.

Lemma 3.12. Let $n \geq 2$ be such that $d\left(x_{n}, x_{n+1}\right)<d\left(x_{n}, x_{n-1}\right)$. Then for all $q \geq n+1,2 d\left(x_{n}, x_{n-1}\right) \geq$ $d\left(x_{n}, S x_{q}\right)$.

Proof. Let $q \geq n+1$ and put $v:=p_{q} \geq p_{n+1}$. Then $x_{q}=y_{v}$ and the conclusion follows by Lemma 3.9.

Lemma 3.13. We have that $\lim _{n \rightarrow \infty} d\left(x_{n}, S x_{n}\right)=0$.

Proof. It follows immediately from Lemma 3.7 and the fact that for all $k, 2 d\left(x_{k}, x_{k+1}\right) \geq d\left(x_{k}, S x_{k}\right)$.

Thus, by the establishing properties of $S, p \in F i x(S)=F i x(T)$.

Proposition 3.14. The sequence $\left(y_{n}\right)$ converges to $p$.

Proof. Let $\varepsilon>0$. We want an $N$ such that for all $n \geq N, d\left(y_{n}, p\right) \leq \varepsilon$. Let $k$ be such that $d\left(x_{k}, p\right) \leq \varepsilon$ and put $N:=p_{k}$. Then the conclusion follows by Lemma 3.5.

We may also show that $\left(y_{n}\right)$ is Cauchy without referring to its limit, by first proving the following analogue of Lemma 3.10.

Lemma 3.15. Let $n \geq 2$ be such that $d\left(x_{n}, x_{n+1}\right)<d\left(x_{n}, x_{n-1}\right)$. Then for all $v \geq p_{n+1}, 2 d\left(x_{n}, x_{n-1}\right) \geq$ $d\left(x_{n}, y_{v}\right)$.

Proof. If $v=p_{n+1}, y_{v}=x_{n+1}$, so the conclusion holds by our hypothesis. Take an $v \geq p_{n+1}$ and assume the conclusion holds for all $l \in\left[p_{n+1}, v\right]$. We want to prove it for $v+1$. Since $v+1 \geq p_{n+1}+1$, $m_{v+1} \geq n+2$. Set $i:=m_{v+1}-1 \geq n+1$. Then

$$
y_{v+1}=\frac{z_{i v}+S y_{v}}{2} .
$$

Set

$$
s:=\min \left\{k \geq 0 \mid m_{v-k} \leq i\right\} .
$$

Since $m_{p_{n+1}}=n+1 \leq i$, we have that $s \leq v-p_{n+1}$. We also have that $m_{v-s} \leq i$ and $m_{v-s+1}>i$ (noting, for the edge case $s=0$, that $m_{v+1}>i$ ). Since $m_{v-s} \geq m_{v-s+1}-1>i-1$, we have that 
$m_{v-s}=i$. By the minimality of $s$, for all $k \in[0, s), m_{v-k}>i$, i.e. for all $k \in(0, s], m_{v-s+k}>i$, so for all $k \in[0, s], z_{i(v-s+k)}=z_{i(v-s)}=z_{m_{v-s}(v-s)}=y_{v-s}$. In particular, for $k:=s, z_{i v}=y_{v-s}$. Put $l:=v-s$. Then, since $s \in\left[0, v-p_{n+1}\right], l \in\left[p_{n+1}, v\right]$. By the induction hypothesis, we have that

$$
2 d\left(x_{n}, x_{n-1}\right) \geq d\left(x_{n}, y_{l}\right)=d\left(x_{n}, z_{i v}\right) .
$$

In addition, by Lemma 3.9, we have that

$$
2 d\left(x_{n}, x_{n-1}\right) \geq d\left(x_{n}, S y_{v}\right),
$$

So

$$
d\left(x_{n}, y_{v+1}\right) \leq \frac{1}{2} d\left(x_{n}, z_{i v}\right)+\frac{1}{2} d\left(x_{n}, S y_{v}\right) \leq 2 d\left(x_{n}, x_{n-1}\right)
$$

Proposition 3.16. The sequence $\left(y_{n}\right)$ is Cauchy.

Proof. Let $\varepsilon>0$. We want an $M$ such that for all $n, m \geq M, d\left(y_{n}, y_{m}\right) \leq \varepsilon$. Put $\delta:=\frac{\varepsilon}{4}$. By Lemma 3.7, there is an $N$ such that for all $k \geq N, d\left(x_{k}, x_{k+1}\right) \leq \delta$.

Case I. We have that $x_{N}=x_{N+1}=x_{N+2}$.

Then, by Lemma 3.8 , for all $j \geq p_{N+2}, y_{j}=x_{N}$, so we may take $M:=p_{N+2}$.

Case II. There is a $k \in\{N, N+1\}$ such that $x_{k} \neq x_{k+1}$.

Put $\rho:=d\left(x_{k}, x_{k+1}\right)>0$. Again, by Lemma 3.7, there is a $p \geq k+1$ such that $d\left(x_{p}, x_{p+1}\right) \leq \frac{\rho}{2}$, so there is an $s \in[k+1, p]$ such that $d\left(x_{s}, x_{s+1}\right)<d\left(x_{s}, x_{s-1}\right)$. Thus, by Lemma 3.15, for all $v \geq p_{s+1}$, $2 d\left(x_{s}, x_{s-1}\right) \geq d\left(x_{s}, y_{v}\right)$.

On the other hand, since $s \geq k+1, s-1 \geq k \geq N$, so $d\left(x_{s}, x_{s-1}\right) \leq \delta$ and for all $v \geq p_{s+1}$, $d\left(x_{s}, y_{v}\right) \leq 2 \delta$.

Put $M:=p_{s+1}$. Let $n, m \geq M$. Then $d\left(y_{n}, y_{m}\right) \leq d\left(x_{s}, y_{n}\right)+d\left(x_{s}, y_{m}\right) \leq 4 \delta=\varepsilon$.

Since $X$ is complete, $\left(y_{n}\right)$ is convergent and we again denote its limit by $p$. Since $\left(x_{n}\right)$ is a subsequence of $\left(y_{n}\right),\left(x_{n}\right)$ also converges to $p$, and so, as before, $p \in F i x(S)=F i x(T)$. If we do not want to use this detour via the convergence of $\left(x_{n}\right)$, we must prove the following analogue of Lemma 3.13.

Lemma 3.17. We have that $\lim _{n \rightarrow \infty} d\left(y_{n}, S y_{n}\right)=0$.

Proof. Let $\varepsilon>0$. We want an $M$ such that for all $n \geq M, d\left(y_{n}, S y_{n}\right) \leq \varepsilon$. Put $\delta:=\frac{\varepsilon}{4}$. By Lemma 3.7, there is an $N$ such that for all $k \geq N, d\left(x_{k}, x_{k+1}\right) \leq \delta$.

Case I. We have that $x_{N}=x_{N+1}=x_{N+2}$.

Then, by Lemma 3.8, for all $j \geq p_{N+2}, y_{j}=x_{N}$ and $S y_{j}=S x_{N}=x_{N}=y_{j}$, so we may take $M:=p_{N+2}$.

Case II. There is a $k \in\{N, N+1\}$ such that $x_{k} \neq x_{k+1}$.

Put $\rho:=d\left(x_{k}, x_{k+1}\right)>0$. Again, by Lemma 3.7, there is a $p \geq k+1$ such that $d\left(x_{p}, x_{p+1}\right) \leq \frac{\rho}{2}$, so there is an $s \in[k+1, p]$ such that $d\left(x_{s}, x_{s+1}\right)<d\left(x_{s}, x_{s-1}\right)$. Thus, by Lemma 3.15, for all $v \geq p_{s+1}$, $2 d\left(x_{s}, x_{s-1}\right) \geq d\left(x_{s}, y_{v}\right)$. In addition, by Lemma 3.9 , for all $v \geq p_{s+1}, 2 d\left(x_{s}, x_{s-1}\right) \geq d\left(x_{s}, S y_{v}\right)$.

On the other hand, since $s \geq k+1, s-1 \geq k \geq N$, so $d\left(x_{s}, x_{s-1}\right) \leq \delta$ and for all $v \geq p_{s+1}$, $d\left(x_{s}, y_{v}\right)+d\left(x_{s}, S y_{v}\right) \leq 4 \delta$.

Put $M:=p_{s+1}$ and let $n \geq M$. Then $d\left(y_{n}, S y_{n}\right) \leq d\left(x_{s}, y_{v}\right)+d\left(x_{s}, S y_{v}\right) \leq 4 \delta=\varepsilon$. 


\section{Acknowledgements}

This work has been supported by the German Science Foundation (DFG Project KO 1737/6-1) and by a grant of the Romanian Ministry of Research, Innovation and Digitization, CNCS/CCCDI - UEFISCDI, project number PN-III-P1-1.1-PD-2019-0396, within PNCDI III.

\section{References}

[1] A. D. Aleksandrov, A theorem on triangles in a metric space and some of its applications. Trudy Math. Inst. Steklov 38, 4-23, 1951.

[2] M. Bridson, A. Haefliger, Metric spaces of non-positive curvature. Grundlehren der Mathematischen Wissenschaften vol. 319, Springer-Verlag, 1999.

[3] J. A. Clarkson, Uniformly convex spaces. Trans. Amer. Math. Soc. 40, no. 3, 415-420, 1936.

[4] K. Goebel, W. A. Kirk, A fixed point theorem for asymptotically nonexpansive mappings. Proc. Amer. Math. Soc. 35, 171-174, 1972.

[5] K. Goebel, W. A. Kirk, Iteration processes for nonexpansive mappings. In: S. P. Singh, S. Thomeier, B. Watson (eds.), Topological Methods in Nonlinear Functional Analysis (pp. 115-123), Contemporary Mathematics 21, AMS, 1983.

[6] K. Goebel, S. Reich, Uniform convexity, hyperbolic geometry, and nonexpansive mappings. Monographs and Textbooks in Pure and Applied Mathematics 83, Marcel Dekker, Inc., New York, 1984.

[7] M. Gromov, Hyperbolic groups. In: S. M. Gersten (ed.), Essays in group theory. Math. Sci. Res. Inst. Publ., 8, Springer, New York, pp. 75-264, 1987.

[8] S. Kaniel, Construction of a fixed point for contractions in Banach space. Israel J. Math. 9, 535-540, 1971.

[9] W. A. Kirk, Krasnosel'skii iteration process in hyperbolic spaces. Numer. Funct. Anal. and Optimiz. 4, 371-381, 1982 .

[10] U. Kohlenbach, Some logical metatheorems with applications in functional analysis. Trans. Amer. Math. Soc. 357, no. 1, 89-128, 2005.

[11] U. Kohlenbach, Applied proof theory: Proof interpretations and their use in mathematics. Springer Monographs in Mathematics, Springer, 2008.

[12] U. Kohlenbach, L. Leuştean, A quantitative mean ergodic theorem for uniformly convex Banach spaces. Ergodic Theory Dynam. Systems 29, 1907-1915, 2009.

[13] U. Kohlenbach, L. Leuştean, Asymptotically nonexpansive mappings in uniformly convex hyperbolic spaces. Journal of the European Mathematical Society 12, 71-92, 2010.

[14] L. Leuştean, A quadratic rate of asymptotic regularity for CAT(0)-spaces. Journal of Mathematical Analysis and Applications 325, 386-399, 2007.

[15] L. Leuştean, Nonexpansive iterations in uniformly convex $W$-hyperbolic spaces. In: A. Leizarowitz, B. S. Mordukhovich, I. Shafrir, A. Zaslavski (eds.), Nonlinear Analysis and Optimization I: Nonlinear Analysis (pp. 193-209), Contemporary Mathematics 513, American Mathematical Society, 2010.

[16] L. Leuştean, An application of proof mining to nonlinear iterations. Annals of Pure and Applied Logic 165, 1484-1500, 2014.

[17] J. J. Moloney, Some fixed point theorems. Glas. Mat. Ser. III 24, no. 1, 59-76, 1989. 
[18] J. J. Moloney, Construction of a sequence strongly converging to a fixed point of an asymptotically nonexpansive mapping. J. Math. Anal. Appl. 182, no. 3, 589-593, 1994.

[19] S. Reich, I. Shafrir, Nonexpansive iterations in hyperbolic spaces. Nonlinear Analysis 15, 537-558, 1990.

[20] W. Takahashi, A convexity in metric space and nonexpansive mappings. I. Kodai Math. Sem. Rep. 22, 142-149, 1970. 